\title{
Preface: Special Issue in Honor of Harvey Scher's 80th Birthday
}

\author{
Brian Berkowitz ${ }^{1}$. Martin Blunt ${ }^{2}$. \\ Harvey Scher $^{1}$
}

Published online: 22 October 2016

(C) Springer Science+Business Media Dordrecht 2016

\section{Foreword}

It is with great pleasure that we introduce this special issue of Transport in Porous Media, on "Flow and Transport in Porous Media," to honor Harvey Scher on the occasion of his 80th birthday.

Harvey Scher has had-and continues to have-a remarkable career. After completing his Ph.D. in physics at Syracuse University in 1963, he established his research career, as a postdoc, with his first paper on the electronic properties of metals. He followed as a Fellow at Bell Telephone Laboratories (1965-1967), and subsequently developed three careers, establishing pioneering research contributions in all of them.

First, Harvey carried out research at the Xerox Webster Research Center (1967-1983), rising to the position of Principal Scientist. In this career, he managed one of the premier research groups in Xerox, leading a broad research program in electronic properties of molecular/polymeric systems, and developing new theoretical approaches to electronic transport in condensed matter, including the theory of dispersive transport in disordered solids.

Second, he was a Senior Research Associate and Branch Manager at BP (British Petroleum) in Ohio (1983-1993), as well as an Adjunct Professor in the Department of Physics at Case Western Reserve University (1984-1993). During this period at BP, Harvey created and developed the Applied Physics Group, establishing an outstanding small group of theoretical and experimental physicists and physical chemists, and guiding research programs in materials, physics of flow through porous media, complex fluids, and laser spectroscopy (to study materials and catalysis).

$凶$ Harvey Scher

Harvey.Scher@weizmann.ac.il

1 Department of Earth and Planetary Sciences, Weizmann Institute of Science, 7610001 Rehovot, Israel

2 Department of Earth Science and Engineering, Imperial College, London SW7 2AZ, UK 
Third, since 1994, Harvey has served as a Special Contract Professor at the Weizmann Institute of Science, developing a research program in hydrogeology with an emphasis on applying new theoretical methods to model key processes of water/chemical transport.

In honor of Harvey's 80th birthday, this special issue contains a series of papers that touch on various aspects of transport-fluid, mass and heat-in porous media, all of which are related to modeling approaches and scientific frameworks that are connected closely to Harvey's contributions from his third career.

We take this opportunity to thank Harvey for his deep insights and far-reaching, scholarly contributions to our particular field, through publications, mentoring, teaching and participation in workshops. He has inspired generations of scientists and engineers. We look forward to continued interactions together in the years to come!

Brian Berkowitz

Guest Editor

Martin Blunt

Editor-in-Chief

\section{Preface}

This is part 2 of my Festschrift ${ }^{1}$ and I am grateful for this honor to those of my colleagues who dedicated time and effort to it. I started my first Festschrift with the old adage of Satchel Paige, "Don't look back, something may be gaining on you." I concluded it with what may be gaining on me is time past. Instead of being wary of the accumulation of time, I am eager to share this loose chronology of my more recent science activities with friends and colleagues. Interestingly, part 2 coincides with a departure in a number of ways in my life and career.

I took early retirement in 1993 at age 57 from BP Research and immigrated to Israel with my wife Audrey and young daughter Dena (two adult children Michele and Joel remained in the USA). I had to adjust to a different culture and a different language. An important similarity was working in research at the Weizmann Institute of Science (WIS), a large research establishment covering many areas of biological and physical science and mathematics. The bulk of my career in the USA was in the basic science area in multidiscipline institutional laboratories of major corporations: Bell Telephone Laboratories, Xerox Research, BP Research. In an historic aside, all three of these research centers have been basically transformed or eliminated. This fact emphasizes the change for me at WIS in that I returned to an academic environment like the one where I started my career as a postdoc of the eminent physicist Prof. Theodore Holstein.

One can easily discern that the bracketed decade of the 1990s (1989-2000) was a real transition period for me; it was closing areas I had invested years in and starting new ones (with some overlap). There were 23 published papers in a wide variety of topics and journals in that decade. Some of the topics included: Molecular theory of geminate recombination, Hopping transport in disordered systems, Geometric dispersion and unstable flow in porous media, Mechanics modeling using a spring network, Brittle fracture in disordered materials, Time-scale invariance in transport and relaxation, Simulation and theory of two-phase flow in porous media, On characterization of anomalous dispersion in porous and fractured media, Pore-level modeling of wetting, Time-dependent damage evolution and failure in materials, On the structure and transport processes in the capillary fringe of phreatic aquifers, Controlling spatiotemporal chaos in a realistic El Nino prediction model, Anomalous transport in

${ }^{1}$ H. Scher, J. Phys. Chem. B 104(16), 3768 (2000) Reminiscences, Entire volume Harvey Scher Festschrift. 
random fracture networks, On the drift mobility of a molecular polaron in the presence of Coulomb traps. The decade was wrapped up with two experiments (and interpretations) on very different scales-anomalous transport in laboratory-scale, heterogeneous porous media, and field observations of a capillary fringe before and after a rainy season (I participated in the field in the latter set of measurements with D. Ronen). The main collaborators during this time were Prof. William A. Curtin, Ecole Polytechnique Federale de Lausanne (9 papers), Prof. Brian Berkowitz, WIS (6 papers), and Prof. Martin J. Blunt, Imperial College, London, and now editor-in-chief of this journal TiPM (4 papers). The journals ranged from Physical Review to Water Resources Research (although an A.I.P journal, I never heard of it until I started publishing in it) and included four Physical Review Letters, three of them each on a very different topic. The main collaborator that emerged from this decade is Brian Berkowitz with a total up to this point of 36 joint published papers with a real impact on the TiPM field. How did that get started?

The string that ties almost all of this work into a package with common features is disorder in its various guises. I didn't begin in this mode. My first paper, co-authored with Ted Holstein, was on many-body physics: high-frequency cyclotron resonance in an electron-phonon gas. It was published in the Physical Review, and it earned for me a Bell Labs Fellowship. At Bell Labs, I met Mel Lax and in his office one day I spotted a preliminary draft of a problem essentially involving disorder-impurity conduction in semiconductors at low temperatures. He invited me to work on this problem with him and I continued with it after I joined the rapidly growing research laboratory at Xerox Corporation. All the photoconducting imaging materials used in a Xerox machine were disordered, and I thought impurity conduction was a good model system. I was making headway on a self-consistent calculation of diffusion, and as I explained it to Prof. Elliot Montroll, a Xerox consultant, I asked him whether random walks could be applied to this problem. He simply referred me to his paper with George Weiss. I read it, and in the last section the authors generalized the random walk to include random times between steps drawn from a pdf $\psi(t)$. I later called this CTRW (continuous time random walk) and saw that one could use it to calculate $P(\mathbf{s}, t)$, the probability to find the walker on site $\mathbf{s}$ at time $t$. This was precisely what I was looking for; I needed the onesided Fourier transform of $P(\mathbf{s}, t)$. I wrote a theory paper co-authored with M. Lax which has garnered now nearly 1200 citations-not bad for a pure theory paper.

The prize for me, however, was calculating the impurity conduction in n-type $\mathrm{Si}$-there were lots of data with the key parameters known. The experiments showed that the ac conductivity $\sigma(\omega)$ was proportional to $\omega^{\alpha}$ over a large range of $\omega$ (frequency) with $\alpha=0.8$. The challenge was to derive such $\omega$-dependence and for a typical range of impurity concentration $c_{P}$ obtain 0.8 . Everything depended on $\psi(t)$. The whole digression here was to emphasize this point. If $\psi(t)$ is a smooth function with a finite mean time $\tau$, then the CTRW approaches a regular RW as $t / \tau \gg 1$. If $\tau$ doesn't exist or is much larger than the duration time of the measurement, we have the basis for an unusual RW. The diffusion mechanism for impurity conduction is electron transfer from $P \rightarrow P^{+}$in the $c_{P}$ range when the electrons are localized in $P$ atomic orbitals. In this $c_{P}$ range, one can set up a detailed physical model for $\psi(t)$ : electron transfer or hopping among a random distribution of sites. The model was accomplished, and the ensemble average over all the site configurations at a fixed $c_{P}$ was calculated exactly. The resultant $\psi(t)$ contained all the features needed to derive anomalous transport in later applications, using parameterized $\psi(t)$ with these same features, e.g., $t^{-1-\beta}$ for the algebraic-like region. On a $\log (\psi(t))-\log (t)$ plot, these features are discerned as the total shape dependence on $c_{P} R_{B}^{3}$, the measure of disorder ( $R_{B}$, the Bohr radius), an algebraic-like behavior over decades of $\log (t)$ and a "cutoff" of this behavior at $\operatorname{large} \log (t)$. The theory accounted for all the data including the high $c_{P}$ observation of a $\omega^{\alpha} \rightarrow$ dc conductivity $\sigma(0)$ 
transition, a first theoretical result. Hence, with a definite physical model, one established the root of the $\psi(t)$ for ensemble averaged disordered systems. In subsequent application to transient photocurrents in amorphous semiconductors and molecularly doped polymers, anomalous transport was discovered. In that context, in retrospect, impurity conduction is a rare example of a problem containing an anomalous diffusion mechanism (a sequence of defined local transits or hops); anomalous transport is ubiquitous.

Fast-forward to the mid-1990s, I was driving from Jerusalem to WIS in Rehovot with Brian Berkowitz and he was describing a puzzling problem in hydrogeology. The dispersion coefficient $D$ of a chemical plume in aquifers and other porous media is not constant but varies systematically with the length or timescale over which transport occurs. Brian went on to say that one strategy to encompass this behavior to is make the dispersion $D$ a time-dependent one, $D(t)$ and to insert it into the conventional ADE (advective-dispersion equation). I said one can't do that. We talked more and that became the basis of our first paper published in Water Resources Research in 1995.

In my office at WIS in the course of discussing the need for a non-local-in-time equation for these effects and CTRW and anomalous transport, Brian said, "let's apply this CTRW formalism" to what Brian called non-Fickian problems. I was skeptical at first but he was right. I was concerned how we would generate $\psi(t)$ for this case. I was comfortable with the electronic mechanisms of hopping and multiple trapping (called MRMT in this field). Brian suggested we try a 2D random fracture network, a distribution of linear segments. Simulation determined the distribution of steady-state flow $\xi=1 / v$. An analytic form was fit to the distribution and $\psi(t) \rightarrow t^{-1-\beta}$ for large $t$ and $\beta=0.7$. This was another physical model system that produced highly non-Fickian results. It was on a good track, but it was all numerical of course. I always worked with data in the electronic applications. We were very fortunate that Prof. Stephen Silliman was spending a sabbatical with us. All 3 of us collaborated on using his data of a previous experimental study of breakthrough curves (BTCs) in a heterogeneous mix of blocks of fine sand in a matrix of coarse sand. The BTCs were measured at 3 electrode positions L and the agreement of all 3 BTCs with the theoretical curves at different $\mathrm{L}$ and the same value of $\beta=0.87$ was excellent. We published a full study in WRR (including ADE fits which were not adequate). I was satisfied; BTCs became our staple study. In the transport studies in the electronic field, mainly transient photocurrents, one could only measure moments of the transiting plume (of electrons). In this hydrogeological field, one could measure the passage into an absorbing plane of the entire chemical plume. In fact, one could observe dye tracer plumes in transit.

As I moved into the twenty-first century, I was stimulated to increase my palette of experimental studies. I took Danny Ronen's advice to ascertain the structure of the capillary fringe of groundwater. For me, it was back to my interests in two-phase flow in porous media. Now, the non-wetting fluid would be air instead of oil. I was working on infiltration with Martin Blunt. So I said, "Martin let's turn gravity $180^{\circ}$," the water from the surface of the aquifer moves up in the disordered porous medium by capillary forces against gravity. Martin ran the models and Danny and I would see if it corresponded to reality. We rented a truck and crew to provide a Geoprobe measurement. We worked in an orchard near the coast in the Western Galilee. We probed the fringe at a number of locations and found the model fit well. It contradicted the textbook version. It was fun arguing with the referees when we published the first of our 2 papers in TiPM (before Martin became editor-in-chief), the other in the Journal of Contaminant Hydrology. Martin and I unfortunately didn't finish the infiltration studies, but the fringe was fine.

Brian and I were on a roll, we declared our probabilistic approach in a paper in TiPM and continued to concentrate on generating and analyzing laboratory experimental data, as well 
as field observations (jointly with G. Kosakowski a postdoc). I want to pause at this point in our burgeoning research activities and insert that we had excellent students and postdocs. The ones that I collaborated with are: G. Margolin, M. Dentz, A. Cortis, D. Holder, Y. Edery plus wonderful outside colleagues (Brian has an additional list). An important part of a study showing the interconnected aspects of using CTRW to model transport was carried out by Marco Dentz and Andrea Cortis. They demonstrated that the results of solving the pde form of CTRW (using what was dubbed the CTRW Toolbox, made freely available) and a particle tracking with $p(\mathbf{s}) \psi(t)$ generating the particle displacement and local transit time at each step (CTRW-PT) completely coincided. Later we showed that the approximation with the "coupled" $\psi(\mathbf{s}, t) \approx p(\mathbf{s}) \psi(t)$ was a good one providing $p(\mathbf{s})$ is compact, i.e., it does not have an algebraic form like a Lévy walk. The findings with CTRW-PT gave us a lot of confidence to explore new horizons of reactive transport as I shall discuss in my chronology. Brian and I published the work with M. Dentz and A. Cortis in AWR (Advances in Water Research) in 2004. Two years later, the same four authors had an extensive review of the transport work in the Reviews of Geophysics, that included both the theory and comparison with experiment, notably our favorite demo BTC of Scheidegger (1959). During the time this review was being written, I also returned to a topic that culminated for me in the transition decade of the 1990s, namely a spring network model of brittle materials. I worked with E. Aharonov and R. Katsman modeling the compaction response of rocks to compression. Observations showed two types of response to uniaxial compression, one compaction localized in the cap area and another diffusely distributed. The model related these different responses to the extent of disorder in the rock, which was confirmed.

The CTRW-PT approach was proving to be a versatile tool. I called it a hybrid method in that the $p(\mathbf{s}) \psi(t)$ used to generate the space and time particle steps was the result of an ensemble average while the exact particle trajectories had an explicit representation. We launched our intensive program in reactive transport using this method. It has the advantage of modeling the transport of various species in a disordered medium at reasonable length scales while chemical reaction between the species takes place at a pore scale. The fluctuations at the pore-level affect the concentration dynamics at the larger scales. The program was driven by an excellent student of Brian's, Yaniv Edery. The first effort was an analysis of an experiment in the literature by C. M. Gramling et al. of a bimolecular interaction $A+B \rightarrow C$ in a disordered porous medium. It featured a visualization of the evolving spatial profile of $C$ with its subtle tails described well by our CTRW-PT approach. We showed that the asymptotic rate of $C(t)$ production depends on velocity fluctuations. We then analyzed two in-house experiments: One was a study of Brian and his student Olga Singurindy of dissolution and precipitation in dolomite involving a multispecies reaction. We explained what had been a puzzle - spatial oscillations of the precipitate. The other was a new, in the literature, visualization experiment involving $\mathrm{pH}$-induced reactions in a medium packed with acrylic polymer beads. The " $A$ " molecule is the $\mathrm{pH}$ indicator, Congo red, and it could be both a conservative tracer and a reactive one depending on its $\mathrm{pH}$ relative to the background solution. Observing $\mathrm{pH}$ threshold effects is an important part of this study. The combination of intrinsic transport in heterogeneous media and varieties of chemical reactions provides a rich array of data and a testing of models. A review of a large swatch of the reactive transport field, which we have undertaken, makes for a fascinating study and a projection for future research. Compared to the interesting studies of conservative transport in heterogeneous media (e.g., anomalous transport, preferred paths) there is a multiplication of fascinating features in reaction transport in the same media. These features include the interplay of a host of physical, chemical and biological variables that influence the formation of reaction fronts. The subtlety of front formation involves the dynamics of both plume spreading and mixing. 
A good example for future research is well-designed laboratory experiments elucidating the biodegradation of organic solvents typically observed in field measurements. There are many experiments integrated with models that are waiting to be done in the growing, active area of reactive transport.

The CTRW formalism works well for conservative and reactive transport in disordered materials because it delivers the essential feature of a probabilistic distribution of local transition times or internal residence times $\psi(t)$. Let me amplify the last variable and its connection with $\psi(t)$. We recently considered a representative model of a heterogeneous porous medium - a lognormally distributed hydraulic conductivity $(K)$ field. The transport was determined by a Lagrangian particle tracking method. We first established the dominance of preferential pathways across each domain and characterized the statistics of these pathways by forming a particle-visitation weighted histogram, $\mathcal{H}_{w}(K)$. We converted the $\ln (K)$ dependence into cell residence times and demonstrated the equivalence of $\mathcal{H}_{w}(K)$ and $\psi(t)$ and delineated the region of $\mathcal{H}_{w}(K)$ that forms the power-law part of $\psi(t)$. We pinpointed the narrow low $K$ regions, embedded in the preferred paths, which determine the power law. It is the origin of anomalous transport and shows the subtlety of the effect - the transport is carried by the preferred paths, but the encounter with these low $K$ parcels is sufficient to broaden $\psi(t)$. We have recently collaborated in a study of bimolecular reactions in the same system with a similar conceptual picture emerging (see the article by Y. Edery et al. in this issue). The equivalence between these two statistical characterizations of the disorder $\mathcal{H}_{w}(K)$ and $\psi(t)$ is a remarkable result.

A poem by the American Robert Frost speaks of the path not taken. For me that path would be the one continuing my life in the USA. I have no doubt coming to Israel, and pursuing a research career at the Weizmann was the preferred path. The poem ends with

I took the one less traveled by,

And that made all the difference.

My family has thrived and adjusted very well here and mastered Hebrew (not me). My daughter has two lovely children. Alma Mia is $51 / 2$ and Shalem 4; they live a 10-min walk from us in Jerusalem. We love being grandparents and living so close to them. I have so much to be thankful for: first and foremost my lovely wife Audrey joining me on this path enthusiastically together. Again I thank my colleagues for this honor, especially Brian Berkowitz who put a lot of time and effort into pulling this special issue together. Lastly, I thank all who contributed such fine articles to this issue and to Martin Blunt for editing it.

Harvey Scher

Harvey.Scher@weizmann.ac.il 